\title{
Generation of postured voxel-based human models for the study of step voltage excited by lightning current
}

\author{
J. Gao ${ }^{1,2}$, I. Munteanu ${ }^{1,3}$, W. F. O. Müller ${ }^{2}$, and T. Weiland ${ }^{1,2,3}$ \\ ${ }^{1}$ Graduate School of Computational Engineering, Technische Universität Darmstadt, Dolivostraße 15, 64293 Darmstadt, \\ Germany \\ ${ }^{2}$ Institut für Theorie Elektromagnetischer Felder, Technische Universität Darmstadt, Schloßgartenstraße 8, 64289 Darmstadt, \\ Germany \\ ${ }^{3}$ CST - Computer Simulation Technology AG, Bad Nauheimer Straße 19, 64289 Darmstadt, Germany
}

\begin{abstract}
With the development of medical technique and computational electromagnetics, high resolution anatomic human models have already been widely developed and used in computation of electromagnetic fields induced in human body. Although these so called voxel-based human models are powerful tools for research on electromagnetic safety, their unchangeable standing posture makes it impossible to simulate a realistic scenario in which people have a lot of different postures. This paper describes a poser program package which was developed as an improved version of the free-from deformation technique to overcome this problem. It can set rotation angles of different human joints and then deform the original human model to make it have different postures. The original whole-body human model can be deformed smoothly, continuity of internal tissues and organs is maintained and the mass of different tissues and organs can be conserved in a reasonable level.

As a typical application of the postured human models, this paper also studies the effect of the step voltage due to a lightning strike on the human body. Two voxel-based human body models with standing and walking posture were developed and integrated into simulation models to compute the current density distribution in the human body shocked by the step voltage. In order to speed up the transient simulation, the reduced $c$ technique was used, leading to a speedup factor of around 20. The error introduced by the reduced $c$ technique is discussed and simulation results are presented in detail.
\end{abstract}

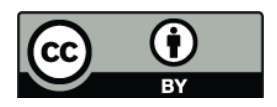

Correspondence to: J. Gao (gao@gsc.tu-darmstadt.de)

\section{Introduction}

Since coming into being in the last century, medical imaging technique has developed rapidly. Nowadays, it allows the construction of digital three-dimensional computational human models based on actual anatomy of individual humans by scanning a real person by means of computer tomography (CT) or magnetic resonance imaging (MRI) techniques. The usefulness of these so-called voxel-based human body models lies in their faithful representation of human anatomy. Until now there are already more than twenty voxel-based human models reported including different ethnicities, ages, sizes, heights and genders (Caon, 2004).

However, most of these voxel-based human models only have their initial standing posture, which significantly limits related research activities including a realistic scenario where postured human bodies must be considered. In order to solve this problem, several deformed voxel-based human models have already been developed, such as the sitting human models developed by Dawson et al. (1999), Allen et al. (2003, 2005), Findlay and Dimbylow $(2005,2006)$ et al.; the deformed human model with outstretched or forward stretched arms developed by Dawson et al. (1999, 2002), Findlay and Dimbylow (2005); and the deformed human models with several different postures developed by Nagaoka and Watanabe (2008). Until now the most popular method used to develop a postured human model is realized by cutting, rotating and re-attaching the limb to the trunk, and then adding manual editing around the joint attachment areas to ensure tissue continuity. However, developing a deformed human model from the heterogeneous voxel model with a manual editing procedure is very time-consuming. Thus it's necessary to develop a software tool which can be used to generate different kinds of postured human models conveniently.

In this paper, we describe a poser software package which was developed to posture the most popular voxel-based 


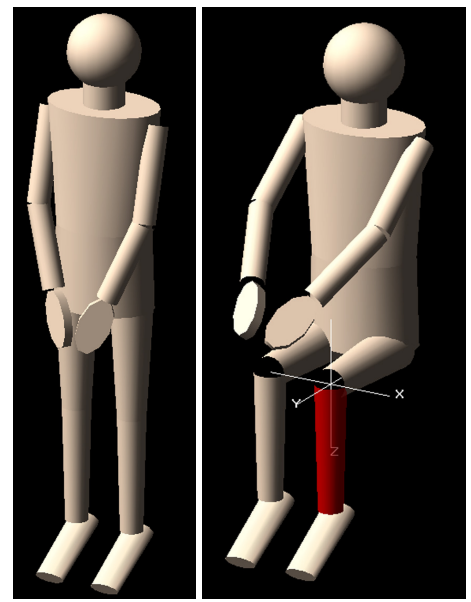

Fig. 1. Simplified human model with initial standing posture and deformed sitting posture, which was designed to define rotation angles of different joints of the human body.

human model built from the Visible Human Project ${ }^{\circledR}$. Compared with other voxel-based human models, this model is even more challenging, because its two arms are originally bent in front of the abdomen and touch the trunk. A segmentation approach was developed to separate arm voxels away from the trunk, which avoids the possible artifacts caused by the simple cutting method. Apart from that deformation of the knee joint was dealt with separately to avoid unreasonable deformation of the femur and tibia, and to rotate the patella into its right position when the knee joint is bent.

\section{Generation of postured voxel-based human models}

\subsection{Voxel-based human model}

The voxel-based human model studied in this paper named "Hugo" (Dipp GmbH, 2010) was built based on the most popular human model from the Visible Human Project ${ }^{\circledR}$ (VHP) funded by the United States National Library of Medicine (Ackerman, 1998). It has already been used for different kinds of applications especially in electromagnetics (Gjonaj et al., 2002). This human model offers a variable voxel resolution ranging from $8 \times 8 \times 8 \mathrm{~mm}^{3}$ to $1 \times 1 \times$ $1 \mathrm{~mm}^{3}$. Each voxel is assigned a unique tissue type, with a total of 31 different tissue types. In the finest resolution, the model consists of approximately 380 million voxels. It represents a 38 years old, male person of $187 \mathrm{~cm}$ height and an approximate weight of $113 \mathrm{~kg}$. Its original posture was lying on an experimental table, similar to an upright standing posture.

\subsection{Workflow of the developed poser program}

The deformation technique used in the poser program was developed based on the free-form deformation technique
(FFD) introduced by Sederberg and Parry in 1986 (Sederberg and Parry, 1986). The FFD technique is a very versatile tool to arbitrarily deform three-dimensional solid geometric models. Through a lattice of movable control points, the FFD technique can deform any kinds of complex geometric model in a free form style. At the same time, the continuity and the topology of the original model can be conserved.

In order to deform the original voxel-based human model as conveniently as possible, a graphical user interface was developed, whose main functionality is described below.

The very first step of the poser program is to import the original voxel dataset file and to display the threedimensional human models based on different tissues and organs. The rendering function of the poser program was realized by the marching cubes algorithm (Lorensen and Cline, 1987), which can extract outer surfaces of different tissues and organs of the voxel dataset. After checking the original human model, the user can switch to a simplified human model (Fig. 1) in order to set the rotation angles of different joints, and, at the same time, to get a general impression about the deformed shape of the postured human model.

After the rotation angles of all considered joints are defined satisfactorily, the deformation calculation can be performed to posture the original human model. Two special issues related to this step are described in more detail in Sects. 2.4 and 2.5. At any point, the user can go back to the simplified human model mentioned above to redefine the rotation angles of some joints and perform deformation calculation again, until a satisfactory result is achieved. In the last step, the postured human body model is meshed and exported as new voxel dataset file.

The outer surface and the internal structure of a sitting human model generated by the poser program are shown in Fig. 2. It can be seen that the outer surface of the whole human body is deformed smoothly, and the continuity of all internal tissues and organs is conserved.

\subsection{Mass conservation}

An important issue for the deformation of biological models is the conservation of their mass. This is especially important for simulations involving the calculation of the Specific Absorption Rate (SAR), whose accuracy depends in an essential way on the tissue mass.

In our poser program, for the sitting human model with $1 \mathrm{~mm}$ resolution, the relative error of the mass of any tissue or organ is below $3.5 \%$ as compared to the original model. The relative error of the whole body mass, which is summation of all tissues and organs, is even below 1\%. This error level is acceptable for most simulations involving human models.

The mass error decreases with increasing resolution $\Delta x$ of the human body model, as shown in Fig. 3. The numerical experiments indicate that the convergence of the whole body mass is of the order $\mathrm{O}\left(\Delta x^{1 / 2}\right)$. 

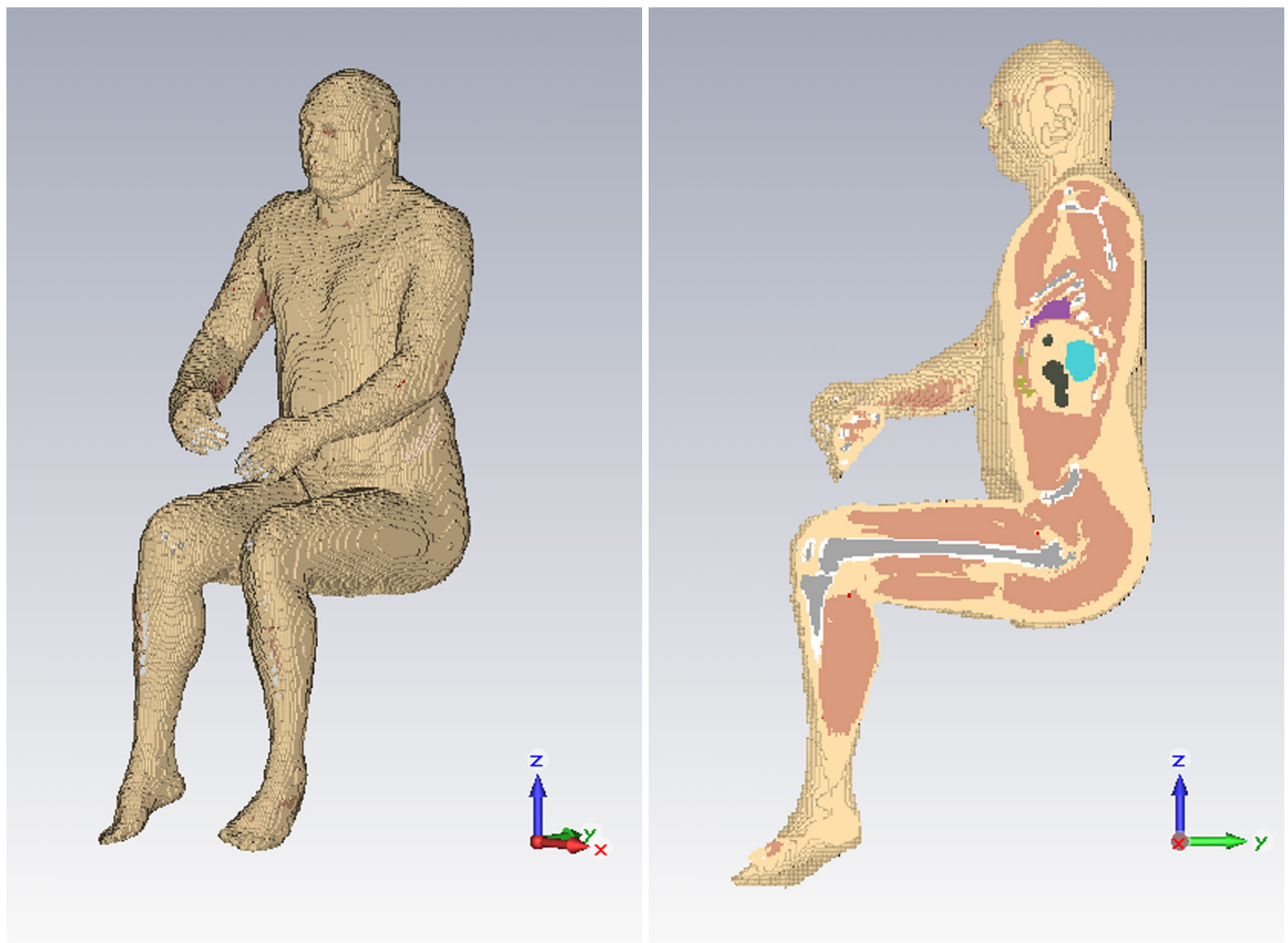

Fig. 2. Outer surface and internal structure of the sitting human model generated from the poser program.

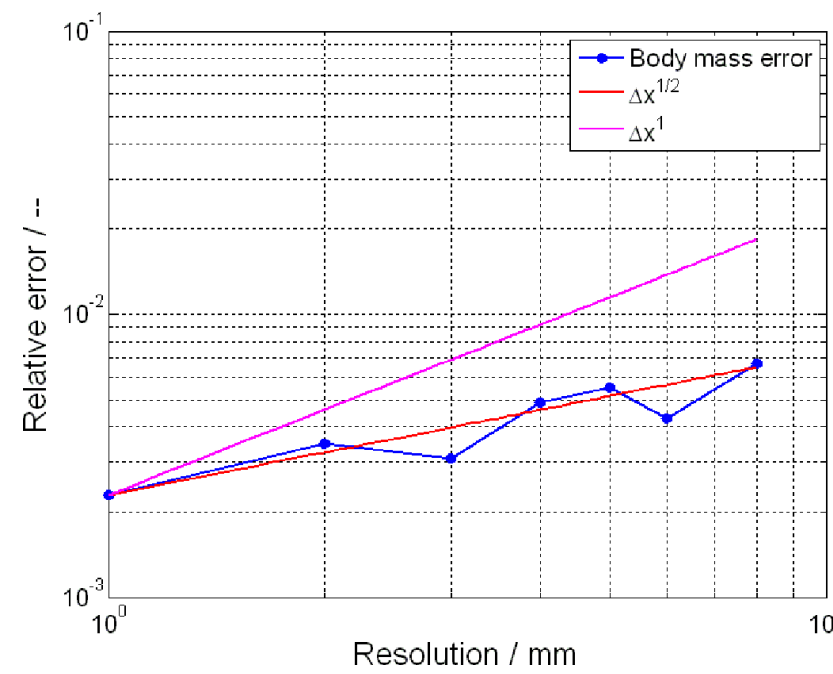

Fig. 3. Relative error of the total body mass, after the movement of the human model to a sitting position. The curves $\Delta x^{1}$ and $\Delta x^{1 / 2}$ are also displayed for reference.

\subsection{Movement of the shoulder joint}

Most voxel-based human models have the arms well separated from the trunk. However, even for this situation a simple cutting procedure must be performed to separate the arm voxels from those of the chest near the armpit, a procedure which is recognized as error-prone (Nagaoka and Watanabe, 2008).

The configuration of the voxel-based human model studied in this paper is even more complicated. Originally its arms are bent in front of the abdomen and they touch the trunk, as shown in Fig. 4. To be able to correctly deform the arm model, all voxels which belong to the arm must be separated from the trunk voxels. Fortunately, in the finest resolution of the voxel dataset, a continuous layer of skin voxels exists around the whole human body model, which can be used for the arm segmentation. The complexity of the human body model near the arm required that the whole arm model be divided into six parts which are dealt with separately in order to get an accurate enough result (Fig. 4).

As an example, the first part of the right arm, shown in Fig. 5a, placed below the right shoulder, can be checked from the center towards the right side as the red arrow in Fig. 5a shows. All voxels found after the skin voxel can be set as arm voxels. The other five parts of the right arm model and their checking directions are listed in respective sub-figures of Fig. 5. They can be dealt with in a similar manner, until all voxels belonging to the arm are separated away from trunk. 


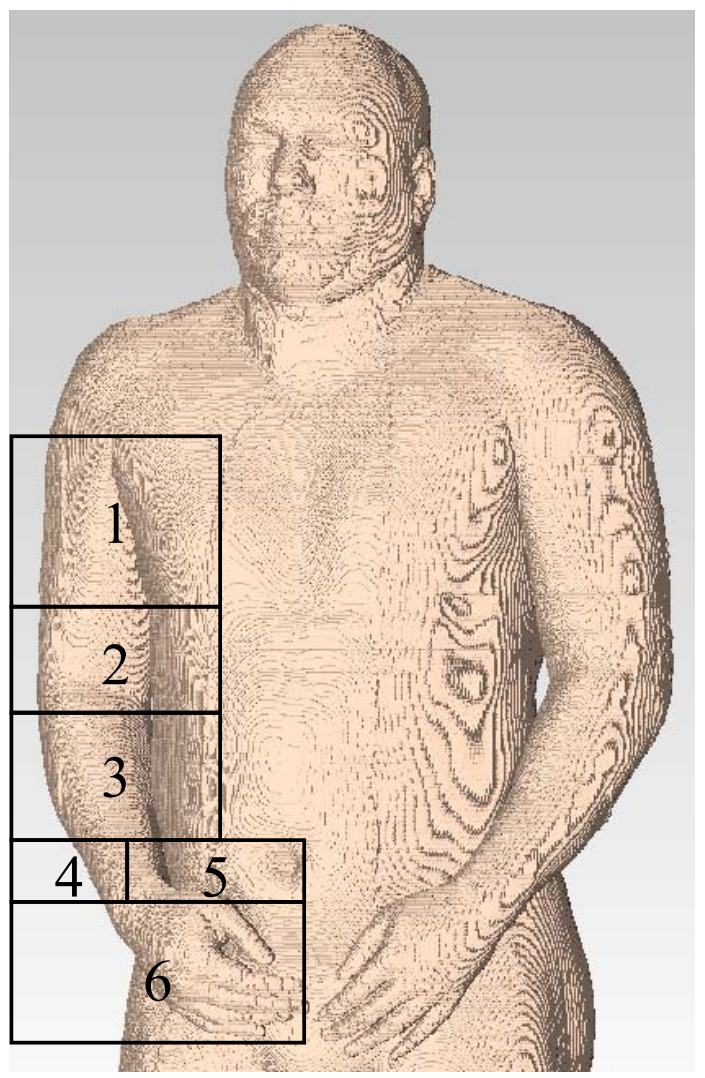

Fig. 4. Initial configuration of the original human model and six parts of the right arm.

\subsection{Movement of the knee joint}

The main advantage of the FFD technique in deforming the voxel-based human model lies in maintaining the continuity of internal tissues and organs, due to the intrinsic "flexible" deformation ensured by the FFD.

However, the anatomically correct movement is complicated in some special joints such as the knee joint. The movement of the knee joint includes not only flexible deformations of the fat, muscle, skin etc., but also rigid body movement and rotation of the skeleton. When the knee joint is bent, the tibia rotates around the bottom surface of the femur, while at the same time, the relative position of the patella with respect to the femur changes. Straightforward application of the FFD technique would also lead to a deformation of the bones and would maintain the patella in an incorrect position. This disadvantage is well known and was previously mentioned in the literature (Nagaoka and Watanabe, 2008).

To circumvent the above mentioned problems, some special measures were taken. They are based on the observation that rigid body movement can also be achieved with FFD, if the FFD control lattice itself is not deformed.

In order to realize all movements of the knee joint mentioned above, three sets of FFD control lattices were defined around the knee joint. The first set is designed to deform the flexible materials such as fat, muscle, skin etc, while the second and third set control lattice are used to rotate the femur, tibia and patella as a whole. The final deformation of the knee joint of the sitting human model generated from the poser program can be seen in Fig. 2, right and consistent with the real anatomic structure.

\section{Application: study on step voltage with postured human models}

\subsection{Introduction to the step voltage caused by lightning current}

When a lightning flash strikes the earth or buildings, the lightning current can flow into the ground through any grounded conductive object, and the discharge current in the ground will cause an electric field in the near area. If a human happens to stand or walk in this region, an electric potential difference between his or her legs exists - the so-called step voltage, whose value is an important reference parameter that must be considered in designing, manufacturing and optimizing lightning protection equipment.

For analysis purposes, the International Electro-technical Commission (IEC) suggests to use following formula to express current wave shape of the short lightning flash stroke (IEC, 2003)

$i(t)=\frac{I}{k} \cdot \frac{\left(t / \tau_{1}\right)^{10}}{1+\left(t / \tau_{1}\right)^{10}} \cdot e^{\left(-t / \tau_{2}\right)}$.

Here, $I=200 \mathrm{kA}$ is the peak current value, $k=0.93$ is a correction factor for the peak value, $\tau_{1}=19 \mu \mathrm{s}$ and $\tau_{2}=485 \mu \mathrm{s}$ are front and tail time constants. The duration of the lightning current signal is defined as $350 \mu$ s.

This time-domain current signal was used as excitation source for all simulations in this section.

\subsection{Simulation models}

In order to study the influence of the step voltage on human's health and to consider the effects of human's posture on the results, two simulation models, one standing and one walking human body model, were used. The original voxel-based human model, with $4 \mathrm{~mm}$ resolution, was deformed into two these postures with the poser program. A step voltage of around $240 \mathrm{kV}$ peak value was used to excite two perfect electric conductive patches placed below the human's feet, which models a human standing around 11 meters away from the point where the lightning strikes. The simulation setup including the walking and the standing human body model is shown in Fig. 6.

A fast Fourier transformation applied to the lightning current signal expressed by Eq. (1) shows that most part of the frequency spectrum of this current signal is below $100 \mathrm{kHz}$. Because of the very low frequency spectrum of the excitation 


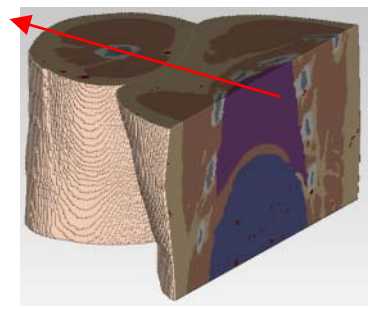

(a) Part I

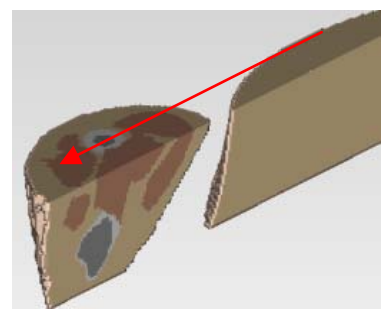

(d) Part IV

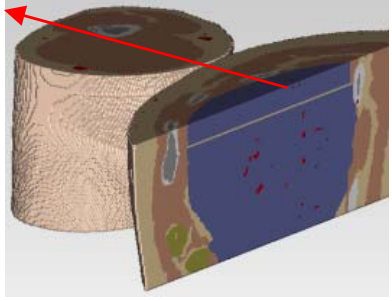

(b) Part II

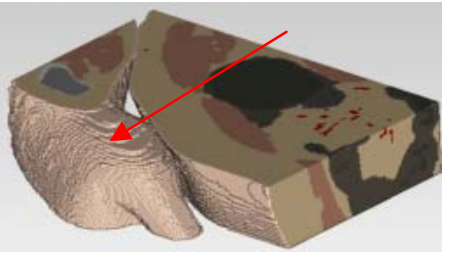

(e) Part V

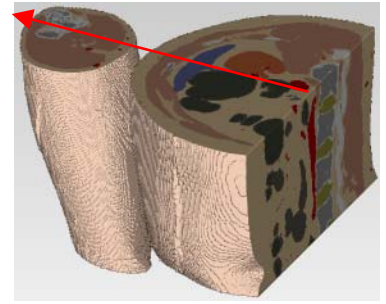

(c) Part III

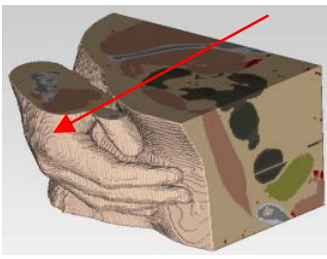

(f) Part VI

Fig. 5. Six parts of the right arm and their checking directions used to separate arm voxels away from the trunk.
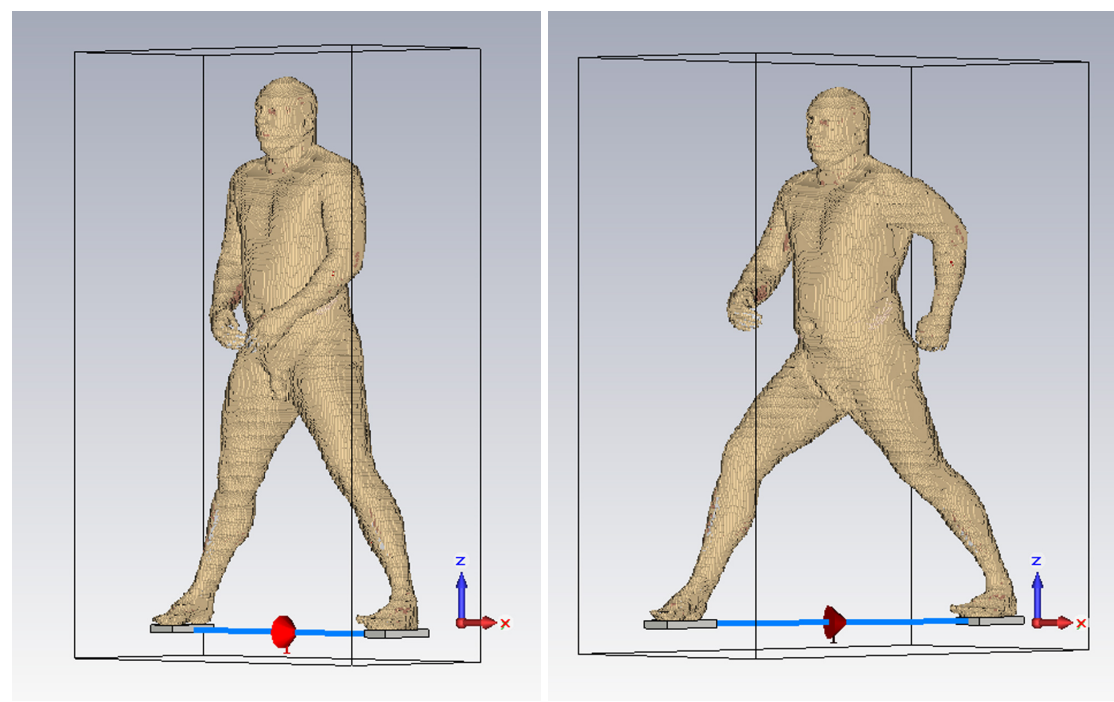

Fig. 6. Simulation models of the step voltage excited by lighting current including human models with standing and walking posture.

signal, the simulation studied here is in principle an electroquasistatic problem. However, in order to take into account the dispersive characteristics of the biological materials, we used a full-wave transient simulator (CST AG, 2010), which can deal with such materials.

The direct use of this explicit full-wave transient simulator for the quasistatic simulation results in a huge number of time steps (around $10^{8}$ ) that need to be simulated and therefore a very long calculation time. The reduced $c$ technique, first introduced by Holland in 1995 to solve a magneto-quasistatic problem (Holland, 1995), was studied to be applied in this simulation. To our knowledge, it has not been previously applied to electro-quasistatic simulations, in which the relative permeability, rather than the relative permittivity, can be increased to mathematically reduce the speed of light, and thus to obtain a much larger stable time step than the value specified by the Courant limit. A detailed error analysis showed that increasing the relative permeability by a factor of 400 leads to a speedup of the simulation around 20 times, while the relative error thus introduced is below $5 \%$ before the peak value of the excitation signal at $t=30 \mu \mathrm{s}$, and below $0.5 \%$ near and after the peak value. 

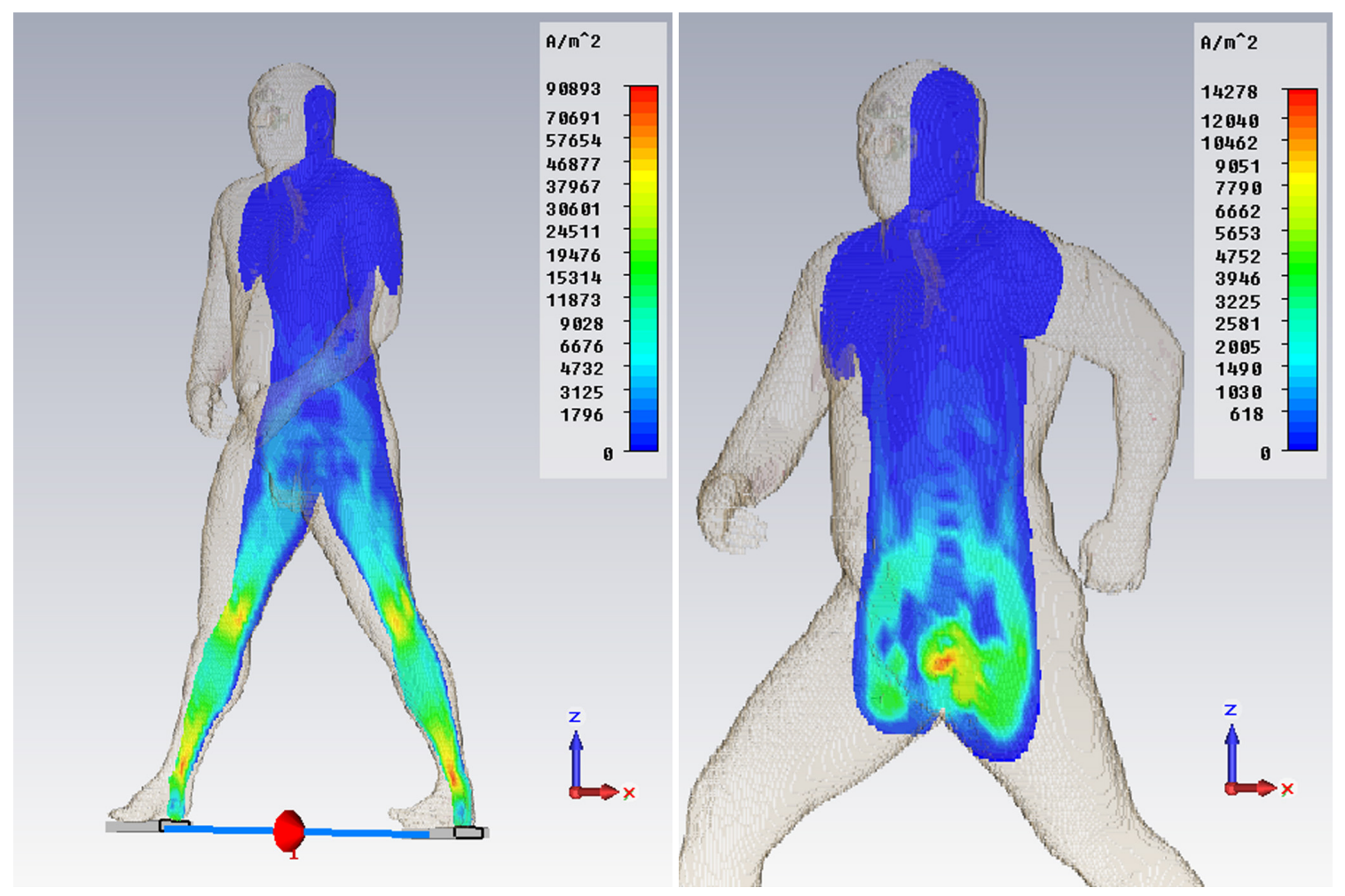

Fig. 7. Current density distribution in the standing and walking human body shocked by the step voltage.

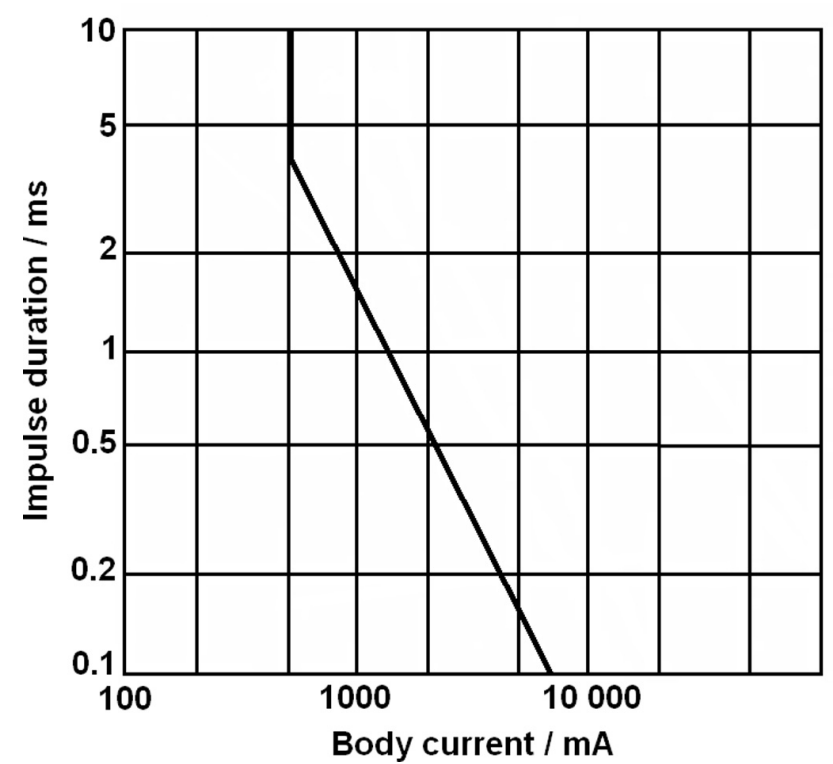

Fig. 8. Threshold of ventricular fibrillation with different body current and impulse duration (IEC, 2006).

\subsection{Simulation results}

Figure 7 shows the current density distribution in the standing and walking human model at $t=30 \mu$ s, which is near the peak value of the lightning current signal. The largest current density appears in the leg muscles, especially near the knee and ankle joints, as well as in the bladder. The current density is comparatively much weaker in bones, due to the much lower conductivity of bone and marrow with respect to that of the muscle, blood and bladder. In the upper part of the human body, which is quite far away from the excitation sources, the current density is lower as well.

As a very important organ in the whole human body, the safety of the heart is always of great concern. The standard 60479 of the International Electrotechnical Commission (IEC) (IEC, 2005) defines the whole body current threshold of ventricular fibrillation of the heart for different excitation impulse durations, as Fig. 8 shows for a signal type which is very similar to a lightning strike. The curve in the figure indicates the probability of fibrillation risks for current flowing along the path from left hand to feet. For a path from left foot to right foot, the value should be divided by a heart current correction factor $F=0.04$ (IEC, 2006). The measurement results from Fig. 8 show that the threshold of the ventricular fibrillation is around $4000 \mathrm{~mA} / 0.04=100 \mathrm{~A}$ effective value for impulse duration $350 \mu \mathrm{s}=0.35 \mathrm{~ms}$ along the path from one foot to the other. Our simulation results here show that the peak value of the whole-body current can reach around $100 \mathrm{~A}$, thus its effective value is much smaller than this value. This means that, due to the very short duration of the excitation signal and to the comparatively long 
distance between the heart and the excitation sources, under the simulation condition above, the whole body current is below the threshold of ventricular fibrillation prescribed by the IEC standard.

Apart from that, the total body current is the almost the same for the standing and the walking human model, which means that the human's posture does not have a big influence on the human body's impedance in frequency range below $100 \mathrm{kHz}$.

\section{Conclusions}

In this paper, we describe a poser program to deform one of the most popular voxel-based human models from the Visible Human Project ${ }^{\circledR}$. The program is based on the free form deformation technique, a versatile and powerful deformation technique in computer graphics. New techniques were developed to segment two arms away from the trunk and for separately dealing with body parts requiring rigid body motion, such as the knee joint, in order to get an anatomically correct postured human body model. The poser program allows the convenient and quick movement of the voxel-based human model, with a minimum of user interaction. The outer surface of the whole human body can be deformed smoothly, and the continuity of internal tissues and organs is ensured. In addition, the relative error of the mass of every tissue and organ between the original and the postured human model can be conserved in a reasonable level.

As an application example, we studied the internal current density distribution in the human body shocked by the step voltage, by using two postured voxel-based human body models. Time-domain calculations were all speeded up around 20 times with low error by using the reduced $c$ technique. Simulation results have shown all dangerous areas of the human body with comparatively higher current density values, which are valuable references for lightning protection engineering.

Acknowledgements. This work is supported by the 'Excellence Initiative' of the German Federal and State Governments and the Graduate School of Computational Engineering at Technische Universität Darmstadt.

\section{References}

Ackerman, M. J.: The visible human project, Proceedings of the IEEE, 86(3), 504-511, 1998.

Allen, S. J., Adair, E. R., Mylacraine, K. S., Hurt, W., and Ziriax, J.: Empirical and theoretical dosimetry in support of whole body resonant RF exposure $(100 \mathrm{MHz})$ in human volunteers, Bioelectromagnetics, 24(7), 502-509, 2003.
Allen, S. J., Adair, E. R., Mylacraine, K. S., Hurt, W., and Ziriax, J.: Empirical and theoretical dosimetry in support of whole body radio frequency (RF) exposure in seated human volunteers at $220 \mathrm{MHz}$, Bioelectromagnetics, 26(6), 440-447, 2005.

Caon, M.: Voxel-based computational models of real human anatomy: a review, Radiat. Environ. Bioph., 42(4), 229-235, 2004.

CST AG: CST - Computer Simulatin Technology, online available at: http://www.cst.com/, accessed: 20 August 2010.

Dawson, T. W., Caputa, K., and Stuchly, M. A.: Numerical evaluation of $60 \mathrm{~Hz}$ magnetic induction in the human body in complex occupational environments, Phys. Med. Biol., 44, 1025-1040, 1999.

Dawson, T. W., Caputa, K., and Stuchly, M. A.: Magnetic field exposures for UK live-line workers, Phys. Med. Biol., 47, 9951012, 2002.

Dipp GmbH: Anatomical volume data sets, online available at: http: //www.vr-laboratory.com/, accessed: 8 November 2010.

Findlay, R. P. and Dimbylow, P. J.: Effects of posture on FDTD calculations of specific absorption rate in a voxel model of the human body, Phys. Med. Biol., 50(16), 3825-3835, 2005.

Findlay, R. P. and Dimbylow, P. J.: FDTD calculations of specific energy absorption rate in a seated voxel model of the human body from $10 \mathrm{MHz}$ to $3 \mathrm{GHz}$, Phys. Med. Biol., 51(9), 2339-2352, 2006.

Gjonaj, E., Bartsch, M., Clemens, M., Schupp, S., and Weiland, T.: High-resolution human anatomy models for advanced electromagnetic field computations, IEEE Transactions on Magnetics, 38(2), 357-360, 2002.

Holland, R.: FDTD analysis of nonlinear magnetic diffusion by reduced c, IEEE Transactions on Antennas and Propagation, 43(7), 653-659, 1995.

IEC: IEC 62305-1: Protection against lighting - Part 1: General principles, International Electrotechnical Commission, 2003.

IEC: IEC 60479-1: Effects of current on human beings and livestock - Part 1: General aspects, International Electrotechnical Commission, 2005.

IEC: IEC 60479-2: Effects of current passing through the human body - Part 2: Special aspects, International Electrotechnical Commission, 2006.

Lorensen, W. E. and Cline, H. E.: Marching cubes: A high resolution 3D surface construction algorithm, in: Proceedings of the 14th annual conference on Computer graphics and interactive techniques (ACM Siggraph 87), 24, 163-169, 1987.

Nagaoka, T. and Watanabe, S.: Postured voxel-based human models for electromagnetic dosimetry, Phys. Med. Biol., 53(24), 70477061, 2008.

Sederberg, T. W. and Parry, S. R.: Free-form deformation of solid geometric models, Proceedings of the 13th annual conference on computer graphics and interactive techniques (ACM Siggraph 86), 20(4), 151-160, 1986. 